\title{
José Rizal, Juana Borrero oder neue Ausweitungen der Moderne
}

Lassen Sie uns gegen Ende unserer Vorlesung einen größeren geographischen Sprung machen, der uns zwar noch im Bereich des spanischen beziehungsweise ehemaligen spanischen Kolonialreiches hält, uns zugleich aber die dritte Phase beschleunigter Globalisierung aus einer anderen, asiatischen Perspektive zu beleuchten erlaubt! Sie wissen bereits, dass wir uns nun den Philippinen zuwenden wollen und einem Schriftsteller, von dem man ohne größere Übertreibung sagen könnte, dass mit seinem literarischen Werk das Spanische als Literatursprache auf den Philippinen nahezu erlosch. So beschäftigen wir uns nun mit einem weitgereisten Autor namens José Rizal sowie einem Roman, der zu den herausragenden Schöpfungen einer Nationalliteratur zählt, welche im nachfolgenden, im 20. Jahrhundert ihre spanische Sprache aufgab.

Mit dem Zusammenbruch des kontinentalen Kolonialreichs Spaniens in Amerika wurden gerade mit Blick auf die Beziehungen zwischen den Philippinen und Mexiko viele der über Jahrhunderte entstandenen Fäden im globalen Webmuster der einstigen iberischen Weltmacht durchtrennt. Über lange Jahrhunderte sorgte die spanische Galeone, welche Manila mit dem neuspanischen Hafen von Acapulco verband, für eine höchst wichtige Verbindung im kulturellen Austausch zwischen den Amerikas und der asiatischen Welt. Weit über die Ausbreitung der Namban-Kunst und die globale Verbreitung asiatischer Paravents hinaus, die in den spanischen Amerikas eine völlig eigene künstlerische Entwicklung erfuhren, ${ }^{1}$ war diese transpazifische Verbindung, die wir kurz im Periquillo Sarniento kennengelernt hatten, von unschätzbarer Wichtigkeit für den globalen Warenverkehr. All diese Handels- und Kulturbeziehungen endeten mit dem Niedergang Spaniens.

Doch sorgte die Tatsache, dass neben den Philippinen auch Kuba, Puerto Rico und zumindest zeitweise der westliche Teil Hispaniolas über weite Strecken des 19. Jahrhunderts im spanischen Kolonialreich verblieben, nicht nur - bei allen insbesondere kulturellen Differenzen und Gegensätzen - für eine Vielzahl struktureller Ähnlichkeiten und Übereinstimmungen. Darüber hinaus erklärt sie verstärkte inter- und transarchipelische Beziehungen zwischen der philippinischen Inselwelt und der (noch spanischen) Karibik. Diese transarchipelischen Relatio-

1 Vgl. Ette, Ottmar: Magic Screens. Biombos, Namban Art, the Art of Globalization and Education between China, Japan, India, Spanish America and Europe in the 17th and 18th Centuries. In: European Review (Cambridge) XXIV, 2 (May 2016), S. 285-296.

Ә Open Access. (C) 2021 Ottmar Ette, publiziert von De Gruyter. (c) BY-NC-ND Dieses Werk ist lizensiert unter einer Creative Commons Namensnennung - Nicht-kommerziell - Keine Bearbeitung 4.0 International Lizenz. https://doi.org/10.1515/9783110703443-035 
nen erweitern unseren transarealen Blickwinkel auf ein 19. Jahrhundert zwischen zwei Welten, den wir in unserer Vorlesung gepflegt haben. Und sie erlauben es uns zugleich, ähnlich wie in unserer Vorlesung $\mathrm{zu}$ den Literaturen des 20. und 21. Jahrhunderts sowie parallel zu unserer Beschäftigung mit der vietnamesischen Schriftstellerin Anna Moï auch im 19. Jahrhundert einer Beziehung nachzugehen, die uns innerhalb der Romanischen Literaturen der Welt nach Asien, zu ihrem vergessenen Kontinent führt.

Als Beleg für den kulturellen Reichtum dieser transpazifischen Relationen mögen Leben und Werk des am 19. Juni 1861 in Calamba auf Luzón in den damals spanischen Philippinen geborenen und am 30. Dezember 1896 als Vordenker der Revolution von spanischen Soldaten hingerichteten José Protacio Mercado Rizal y Alonso Realonda gelten. Nicht zu Unrecht wurde er - etwa von dem mexikanischen Philosophen Leopoldo Zea ${ }^{3}$ - mit dem 1853 in Havanna geborenen José Martí in Verbindung gebracht, dessen bewegtes Leben und weitgespanntes Werk gewiss nicht weniger rastlos und vielgestaltig war als jenes des Autors von Filipinas dentro de cien años. Und schließlich fiel auch Martí 1895 in jenem von ihm entfachten Krieg gegen spanische Truppen, die auch José Rizal als einen der führenden Köpfe der Aufständischen hinrichteten.

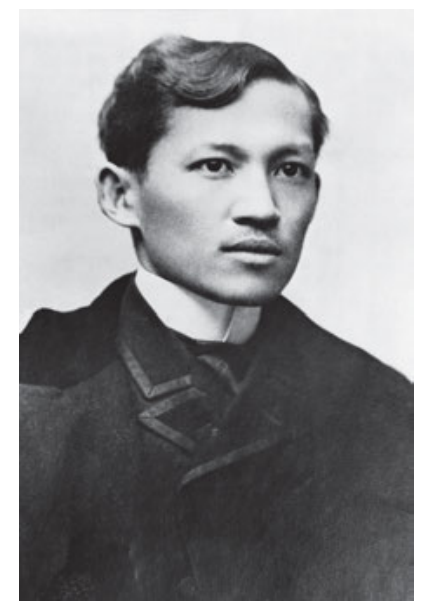

Abb. 83: José Rizal (Calamba City auf Luzon, 1861 - Manila, 1896).

2 Vgl. hierzu den dritten „Aula“-Band in Ette, Ottmar: Von den historischen Avantgarden bis nach der Postmoderne, S. 900-910.

3 Vgl. Zea, Leopoldo: Prólogo. In: Rizal, José: Noli me tangere. Estudio y Cronología Margara Russotto. Caracas: Biblioteca Ayacuch 1976, S. ix-xxx. 
Der Verfasser von Nuestra América hatte lange Jahre seines Lebens in Verbannung und Exil verbracht, die ihn unter anderem nach Spanien, Frankreich und Mexiko, nach Guatemala, Venezuela und in die Vereinigten Staaten sowie die karibische Inselwelt und weitere mittelamerikanische Länder führten. Ähnlich hielt sich José Rizal auf seinen weitgespannten Reisen ebenfalls in Spanien, Frankreich und den USA, aber auch in Deutschland, Österreich und der Schweiz, in Hongkong, Japan, England und Belgien auf, bevor er 1895 vergeblich beantragte, als Arzt die nach Kuba verlegten spanischen Truppen begleiten zu dürfen. Hier zeichnen sich Parallelen zwischen Lebenswegen und Schreibbedingungen ab, die weder aus nur nationalliterarischer noch aus allgemein weltliterarischer Perspektive adäquat erfasst werden können, sondern einer bewusst transareal ausgerichteten Methodologie bedürfen, welche Dynamik und Vektorizität dieser Prozesse ins Zentrum rückt, um adäquat verstanden werden zu können. Denn Martí und Rizal verkörpern als herausragende Repräsentanten ihrer Archipele jene Entwicklungen, die im Zeichen des ersten nicht-europäischen Global Players, im Zeichen der USA der dritten Phase beschleunigter Globalisierung zugerechnet werden dürfen. Beide Schriftsteller gehören zusammen und verdeutlichen die transarchipelische Dimension eines antikolonialen Kampfes, der zugleich auch ein Kampf gegen den aufkommenden Imperialismus der USA war, jener Macht, die als Beute beide Inselwelten wenige Jahre nach dem Tod beider Autoren ihrem rasch expandierenden Machtbereich einverleiben sollte.

Als siebtes von insgesamt elf Kindern in eine wohlhabende Familie geboren, galt Rizal offiziell als chinesischer Mestize, da er in fünfter Generation von einem aus der Provinz Fujian eingewanderten Händler abstammte und seine Mutter eine spanisch-philippinische Mestizin war. Der spätere Nationalheld studierte in Manila zunächst Literatur und Philosophie, nach der Erblindung seiner Mutter dann Augenheilkunde; ein Studium, das er später in Madrid mit Auszeichnung abschloss. Noch bei seinem Aufenthalt in Heidelberg war er Mitarbeiter eines renommierten Ophthalmologen und praktizierte als Arzt. Doch seine Kritik an der Kolonialmacht Spanien, seine Gründung einer Freimaurerloge, seine auf radikale Reformen zielenden politischen Vorschläge sowie vor allem seine kolonialismuskritischen Buchpublikationen brachten ihn in Konflikt mit der spanischen Kolonialmacht.

Das sicherlich bis heute berühmteste Werk José Rizals ist sein 1887 in Berlin auf Spanisch veröffentlichter Roman Noli me tangere. Es ist eine bemerkenswerte Tatsache, dass Martís einziger Roman Amistad funesta (wenn auch postum 1911 im zehnten Band der Werkausgabe von Quesada y Aróstegui) ebenfalls in Berlin erstmals in Buchform erschien. Doch macht diese Anekdote weniger auf Gemeinsamkeiten zwischen beiden Autoren, sondern eher darauf aufmerksam, wie problematisch die spanischsprachigen Verlagsstrukturen zum damaligen 
Zeitpunkt waren. In weit mehr als nur biographischer Hinsicht darf das Werk des philippinischen Nationalhelden einer Literatur ohne festen Wohnsitz zugerechnet werden, der man mit guten Argumenten die kubanische Nationalliteratur insgesamt (und gewiss auch José Martí) zuordnen darf. ${ }^{4}$

Nicht umsonst hatte José Rizal, der auch in deutscher, französischer, englischer und lateinischer Sprache zu lesen und sich auszudrücken wusste, neben seiner Muttersprache, dem Tagalog, in seiner Kindheit ein höchst unvollkommenes Spanisch erlernt, was ihn dazu zwang, anders als ein das Spanische muttersprachlich beherrschender Autor ständig vor einem vielsprachigen Hintergrund an seinen Ausdrucksmöglichkeiten zu arbeiten. Nicht ohne Grund stellte Leopoldo Zea diesen Kampf Rizals um die Sprache seiner Literatur in den geschichtlichen Kontext eines Archipels, das sich nach der Niederlage der spanischen Flotte vor Manila vom Spanischen, der Sprache der kolonialen Unterdrücker, ab- und - unter Stärkung der Position des Tagalog - dem Englischen zuwenden sollte. Es ist, als hätte der philippinische Autor, der sein Lebenswerk in einem translingualen Kontext entfaltete, mit dem Verlust seines Lebens auf den Philippinen zugleich seinen sprachlichen Wohnsitz verloren. Denn die Sprache ihres Nationalhelden steht großen Teilen seines Volkes heute nicht mehr zur Verfügung. ${ }^{5}$

Martí ließ 1895, Rizal im Jahr 1896 das Leben im Kampf gegen eine marode spanische Kolonialherrschaft, deren technologisch weit unterlegene Flotte wenige Jahre später, im Sommer 1898, von der hochgerüsteten Feuerkraft der USPanzerkreuzer vor Santiago de Cuba und Manila erbarmungslos versenkt wurde. Diese Faktenlage soll in unserem Kontext gegenüber der Tatsache zurücktreten, dass sich weder Martí noch Rizal trotz ihres rast- und ruhelosen Kampfes für ihre Heimat auf eine Beschäftigung mit, ihrem' karibischen beziehungsweise philippinischen Archipel beschränkten. Der Spiel- und Bewegungsraum ihres Denkens wie ihrer Reisen war ein unverkennbar transarealer und transarchipelischer, insofern sie auf ihren Wegen wie in ihrem Denken - um mit dem Excipit von Martís Nuestra América zu sprechen - „las islas dolorosas del mar“, ${ }^{6}$ die schmerzerfüllten Inseln des Meeres, stets mit einer globalen Dimension verwoben. Ihren Archipelen kam dabei - in ihren politischen wie vor allem ihren literarischen

4 Vgl. hierzu Ette, Ottmar: Una literatura sin residencia fija. Insularidad, historia y dinámica sociocultural en la Cuba del siglo XX. In: Revista de Indias (Madrid) LXV, 235 (septiembre - diciembre 2005), S. 729-753.

5 Vgl. hierzu Zea, Leopoldo: Prólogo, S. xxix.

6 Martí, José: Nuestra América, S. 23. 
Texten - die Funktion mobiler ZwischenWelten zu: Überkreuzungslinien vielfältigster transkultureller Traditionen.

Keineswegs zufällig setzt das erste von dreiundsechzig Kapiteln des in der Hauptstadt des damaligen Deutschen Reiches auf Spanisch veröffentlichten, auf den Philippinen rasch bekannt gewordenen und alsbald von der spanischen Kolonialverwaltung nach behördlicher sowie akademischer Prüfung verbotenen Romans mit der Darstellung eines großen Abendessens ein. Dieses ist mit viel „couleur locale“ gewürzt und will dem direkt angesprochenen Lesepublikum vor Augen führen, wie derartige Formen der Soziabilität in der „Perla del Oriente“7 abzulaufen pflegten: Die Wichtigkeit von Festessen in asiatischen Kulturen ist noch heute legendär.

In diesem zweifellos kostumbristischen Auftakt von Noli me tangere wird von Beginn an in die nur kurz evozierte tropische Flusslandschaft und ihre erst rudimentär entwickelte Stadtlandschaft (Cityscape) sehr bewusst mit den ,acordes de la orquesta“ und dem „significativo clin-clan de la vajilla y de los cubiertos“8 eine Klanglandschaft, ein Soundscape integriert. Dieser wird wiederum durch eine Landschaft unterschiedlichster Düfte ergänzt, einen spezifischen Smellscape: Die synästhetische Dimension ist von beginn an klar markiert. Gastfreundschaft steht von Anfang an im Zeichen der Orchestrierung globalisierter Sinnesreize und Tischsitten, so dass die lokale Einfärbung vor dem Hintergrund einer weltweiten Zirkulation von Gütern und Gewohnheiten gleichsam translokalisiert wird: Die Philippinen werden literarisch innerhalb weltweiter Verbindungen sinnlich erfahrbar gemacht. Nicht umsonst stellt sich im zweiten Kapitel die Hauptfigur des Romans, der blonde und weitgereiste Crisóstomo Ibarra, kurzerhand selbst der anwesenden philippinischen und spanischen Damenwelt - „unas cuantas jóvenes entre filipinas y españolas“9 - wie den Militärs, Klerikern und anderen Vertretern der Kolonialgesellschaft mit folgenden Worten vor: „Meine Herren, sagte er, in Deutschland gibt es eine Sitte, dass wenn ein Unbekannter einer Versammlung beitritt und niemanden zu seiner Vorstellung findet, er sich selbst mit seinem Namen vorstellen darf. “10 Gesagt, getan! Formen deutscher Soziabilität werden von einem Spanier auf den Philippinen für ein kolonialspanisches und philippinisches Publikum adaptiert.

7 Rizal, José: Noli me tangere Prólogo Leopoldo Zea. Edición y cronología Margara Russotto. Caracas: Biblioteca Ayacucho 1976, S. 8.

8 Ebda.

9 Ebda., S. 9.

10 Ebda., S. 18: „--iSeñores! -dijo-, hay en Alemania una costumbre, cuando un desconocido viene a una reunión y no halla quién le presente a los demás, él mismo dice su nombre y se presenta [...].“ 
Wie auf Ebene der Gastronomie oder der Umgangsformen bleibt die gelungene literarische Inszenierung von Geselligkeit auf den Philippinen keineswegs auf den Archipel oder allein auf die Beziehungen zwischen der asiatischen Inselwelt und der iberischen Halbinsel beschränkt. Denn jenseits der Tatsache, dass der Roman in spanischer Sprache abgefasst ist und vom ersten Kapitel an die peninsulare Variante mit einer von Philippinismen durchsetzten Sprache unterschiedlicher sozialer Kontexte kontrastiert - was auch beinhaltet, dass kürzere Einschübe und Passagen in Tagalog eingefügt werden - greift Noli me tangere bereits im Titel mit seinem Zitat aus dem Lukas-Evangelium auf das Lateinische zurück. Ebenfalls im paratextuellen Bereich geht dem Roman als Motto ein Zitat aus Friedrich Schillers Shakespeares Schatten in deutscher Sprache voraus: Nicht umsonst hat der Schiller-Übersetzer Rizal seinen Roman vielsprachig und translingual angelegt.

Neben den bereits erwähnten Sprachen finden sich aber auch Einsprengsel und Hinweise auf das Französische, Englische und Italienische. Dies verwundert weder angesichts der Vielzahl an Sprachen, die José Rizal sprach, noch mit Blick auf den in der Welt weit herumgekommenen Crisóstomo Ibarra y Magsalin, antwortet dieser doch einem spanischen Mönch, der sich länger in Hongkong aufhielt und daher „Pidgin-English“11 spricht, er liebe die Länder des freien Europa („Europa libre“) und spreche mehrere seiner Sprachen. ${ }^{12}$ Diese sehr bewusst in Szene gesetzte Vielsprachigkeit ist programmatischer Natur: Denn José Rizals Roman führt in seiner eigenen sprachlichen Gestaltung einen weltweiten Archipel der Sprachen vor, wobei er auch auf diesem Gebiet die unübersehbaren Zeichen einer Literatur ohne festen Wohnsitz setzt.

Ohne an dieser Stelle im Kontext der hier behandelten Fragen eine ausführlichere Analyse von Noli me tangere vorlegen zu können, sei doch zumindest betont, welch enorme Rolle von Beginn an dem Haus als fraktalem Muster, als „fractal pattern“13 zukommt. Die ganze Persönlichkeit des Gastgebers, Don Santiago de los Santos alias Capitán Tiago, kommt in jenem Ölgemälde an der Wand zum Ausdruck, das einen „hombre bonito, de frac, tieso, recto, simétrico como el bastón de borlas que lleva entre sus rígidos dedos cubiertos de anillos“14 zeigt. Zugleich konzentriert auch das Haus mit seinem für die Zeit charakteristischen Interieur, seinen weithin berühmten Gelagen, dem ostentativen Konsum seines

11 Ebda., S. 22.

12 Ebda.

13 Vgl. zum fraktalen Muster des Insel-Hauses Ette, Ottmar: Von Inseln, Grenzen und Vektoren. In: ders. / Braig, Marianne / Ingenschay, Dieter / Maihold, Günther (Hg.): Grenzen der Macht Macht der Grenzen. Lateinamerika im globalen Kontext. Frankfurt a.M.: Vervuert 2005, S. 161-167; sowie den Band WeltFraktale (2017) zum Konzept der Literaturen der Welt.

14 Rizal, José: Noli me tangere, S. 9. 
Besitzers und den sich hier begegnenden Menschen wie in einem Brennspiegel die spannungsvolle Welt der kolonialspanischen Philippinen. Die fraktale, eine höchst heterogene Totalität in sich wie in einem „modèle réduit“ (Lévi-Strauss) vereinigende Struktur dieses Hauses leuchtet schon in dessen erster Schilderung auf:

Das Haus, auf das wir anspielen, ist etwas niedrig und von einer nicht sehr korrekten Linienführung: Der Architekt, der es entwarf, mag nicht recht gesehen haben oder alles war die Auswirkung der Erdbeben und Hurrikane, wer wollte dies schon mit Sicherheit sagen. Eine breite Treppe mit grünen Geländern und teils ausgelegten Teppichen führt vom mit bemalten Kacheln geschmückten Eingang oder Portal gesäumt von Blumenampeln und Blumentöpfen, welche auf gesprenkelten chinesischen Absätzen voller fantastischer farbiger Zeichnungen standen, empor zum Hauptgeschoss. ${ }^{15}$

Auf diese Weise bündelt dieses Haus auf den Philippinen, das sich von seiner Umgebung wie eine Insel abhebt, bereits im ersten Kapitel von Rizals Roman wie in einer fraktalen Mise en abyme jenen weltweiten kolonialen und - in der Figur des in seinem Namen nicht zuletzt die spanische und philippinische Herkunft vereinigenden Ibarra y Magsalin - zumindest perspektivisch postkolonialen Bewegungsraum der Kulturen. Dieser drückt sich im Archipel und mehr noch in der weltweit vernetzten Inselwelt der Philippinen aus. Gleichviel, ob es der (koloniale) Architekt oder die Erschütterungen und Wirbelstürme der Zeit waren, welche die Geradlinigkeit dieses Hauses in Frage stellten: Der Archipel der Philippinen steht wie der Archipel der spanischen Karibik am Ausgang des 19. Jahrhunderts vor dem Zusammenbruch einer Kolonialgesellschaft. Letztere sollte im Zeichen der dritten Phase beschleunigter Globalisierung von den gut gebauten Panzerkreuzern der USA nur wenige Jahre später hinweggefegt werden. Das reich geschmückte, aber schiefe Haus legt dies von Beginn des Romans an nahe.

Mehr noch: Im Scheitern der positiv gezeichneten Hauptfiguren des Romans wird auf individueller wie kollektiver Ebene der epochale Schiffbruch des „Desastre“ erkennbar, zu dessen Zuschauer uns der Roman von seiner ersten Zeile an macht. Es handelt sich dabei um einen Schiffbruch, der nicht nur den Untergang der letzten Reste des spanischen Kolonialreichs auf beiden Archipelen, sondern auch des Spanischen auf den Philippinen mit sich bringen wird. Eine neue Zeitrechnung deutet sich an, die - auch wenn sie sich noch einmal den alten Kräften, die auch Rizal selbst ermorden werden, unterwerfen muss - bald all das historisch und dysfunktional werden lässt, was auf beiden Archipelen noch als in sich abgeschlossene und nur auf sich bezogene Insel-Welt geblieben ist.

15 Ebda., S. 8. 
Es ist hier nicht der Ort, die in drastischen Farben vorgetragenen Kritikpunkte am Kolonialsystem, die Verurteilung der allein von spanischen Klerikern vertretenen Kirche, die Anklage gegen den sexuellen Missbrauch philippinischer Frauen und die Verurteilung einer despotischen Kolonialverwaltung nachzuzeichnen, welche den Roman schnell zu einem Opfer der Zensur auf den Philippinen werden ließen. José Rizals Erzähler schilderte in Noli me tangere immer wieder eindrucksvoll die völlig abhängige Situation auch sexuell ausgebeuteter Frauen in einem spanischen Kolonialsystem, welches ihnen eine klar subalterne Stellung zuwies.

Aus diesem Grunde möchte ich mich in dieser Vorlesung wieder der damals noch unter spanischer Kolonialherrschaft stehenden größten der Antillen-Inseln und einer Dichterin zuwenden, mit der ich mich bereits in meiner Vorlesung über die Zusammenhänge von Liebe und Lesen ${ }^{16}$ auseinandergesetzt habe: Juana Borrero. Ihr Zeitgenosse José Martí hatte im Kontext seines lateinamerikanischen Moderne-Entwurfs ein traditionelles, paternalistisches und alle weiblichen Familienmitglieder an die Innenräume des Hauses fesselnde Frauensicht entwickelt, die nach der Lektüre seines am 3. Januar 1887 in La Nación veröffentlichten Artikels selbst von einem Domingo Faustino Sarmiento scharf kritisiert werden konnte. ${ }^{17}$ Dagegen steht die kubanische Lyrikerin und Malerin für ein rebellisches und klar emanzipatorisches Bild der Frau ein. Formulieren wir es anders: Martís in vielerlei Hinsicht revolutionäre Position erstreckte sich nicht auf die Problematik der Geschlechter. Sein Menschenbild blieb Männerbild nicht nur, wo er wie in Nuestra América männliche Protagonisten wählte, sondern gerade auch dort, wo er über die Rolle der Frauen in einer künftigen Gesellschaft sprach. In Martís Diskurs wird das weibliche Subjekt zum Objekt einer männlich modellierten Moderne: Sie spricht nicht, sondern wird gesprochen.

Juana Borrero aber sprach, und sie sprach vehement für sich selbst! Im Februar 1895, zu einem Zeitpunkt also, zu dem José Martí intensiv und ruhelos mit den Vorbereitungen ,seines‘ Krieges und der Invasion Kubas beschäftigt war, notierte eine junge Frau des Nachts in ihr Tagebuch: „Es ist halb eins. Ich habe nicht geschlafen und werde auch nicht mehr schlafen. Gerade habe ich etwas Unerhörtes, Unmögliches, Kühnes gedacht. Hör gut zu, Carlos. Keine zwei

16 Vgl. das Juana Borrero gewidmete Kapitel in Ette, Ottmar: LiebeLesen, S. 552-573.

17 Vgl. hierzu Ette, Ottmar: Geschlechtermodellierungen - Geschlechtergrenzen. José Martí und Juana Borrero. In: Krüger, Reinhard (Hg.): Grenzgänge, Hybride \& Fusionen. Romanistische Beiträge zu polykulturellen Kommunikationsprozessen. Berlin: Weidler Buchverlag 2008, S. 21-39. Dort habe ich versucht, dieses Frauenbild Martís mit seiner Abneigung gegen die moderne Gesellschaft in den USA und mit jenem Gender Trouble in Verbindung zu bringen, dem Judith Butler ihr einflussreiches Buch gewidmet hat. 
Monate und Du gehörst mir oder ich bin tot." ${ }^{18}$ Die 17-jährige Juana Borrero sollte das sich selbst gegebene Versprechen, die dem Mann in Abwesenheit entgegengeschleuderte Herausforderung einlösen und Carlos Pío Uhrbach zu dem ,Ihren“ machen.

Die 1877 geborene Kubanerin stammte aus einer Familie von Literaten, ${ }^{19}$ schrieb bereits mit vier Jahren erste Gedichte und versah mit fünf Jahren die beeindruckende Zeichnung einer Nelke und einer Rose mit dem Titel Romeo y Julieta. ${ }^{20}$ Seit Ende der achtziger Jahre zählte sie als Lyrikerin wie als Malerin zu den großen Hoffnungen der kubanischen Kunst des Jahrhundertendes. Ihre Tagebucheinträge hatten ihr Bild des Geliebten aus der Literatur bezogen und mit dem Bild des von ihr erträumten Mannes in Übereinstimmung zu bringen versucht. ${ }^{21}$ Als sie wenige Monate zuvor den soeben erschienenen Gedichtband Gemelas der beiden kubanischen Lyriker Carlos Pío und Federico Uhrbach erhielt, fragte sie sich bereits vor der Lektüre, die sie sich für die Nacht vornahm: „Wird das ein Gefährte meiner schlaflosen Nächte?“22 Schien damit zunächst das Buch gemeint zu sein, so sprang die Sehnsucht nach einem Gefährten der schlaflosen Nächte schnell auf den älteren der beiden Uhrbach-Brüder über, dessen Verse sie faszinierten und unwiderstehlich anzogen. Das rasch sich bei der jungen Malerin durch die Lektüre hypotypotisch einstellende Bild und Portrait war sicher und unsicher zugleich: „Ein hochfahrendes Gesicht. Carlos müsste bleich sein, ein Kranker.“23 Nicht allein die weiblichen Heldinnen der Romantik und des Jahrhundertendes waren bleich und kränklich: Auch die männlichen Protagonisten weiblicher Liebesträume konnten dies sein. Und die Kubanerin fügte hinzu: „Ist er vielleicht kein Mann?“24

18 Borrero, Juana: Epistolario. 2 Bde. La Habana: Academia de Ciencias de Cuba 1966-1967, hier Bd. I, S. 41: „Son las dos y media. No he dormido ni dormiré. Acabo de pensar algo inaudito, imposible, temerario. Oye Carlos. Antes de dos meses tú serás mío o yo estaré muerta."

19 Vgl. hierzu Cuza Malé, Belkis: El clavel y la rosa. Biografía de Juana Borrero. Madrid: Ediciones Cultura Hispánica 1984.

20 Diese später berühmt gewordene Zeichnung, die auch der Borrero-Biographie der im Exil lebenden kubanischen Lyrikerin Belkis Cuza Malés den Namen gab, findet sich auf Umschlag und Titelseite der beiden Bände des angeführten Epistolario.

21 Vgl. zu Juana Borrero die ausgezeichnete Potsdamer Doktorarbeit von Kern, Anne: Todo o nada. Choreografien der kubanischen Künstlerin Juana Borrero. Potsdam: Potsdam Phil. Diss. 2020.

22 Borrero, Juana: Epistolario, Bd. I, S. 39: „¿Será un compañero de mis insomnios?“

23 Ebda.: „Es un rostro altivo. Carlos debe ser pálido, un enfermo.“

24 Ebda., S. 41: „¿Acaso no es un hombre?“ 


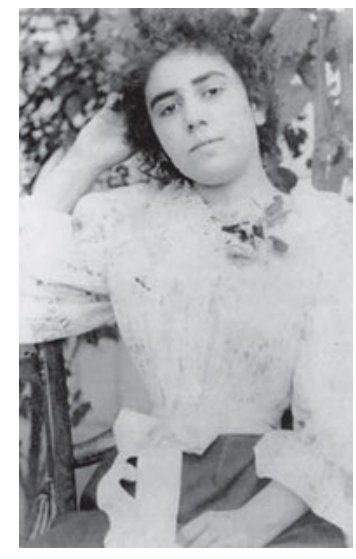

Abb. 84: Juana Borrero

(Havanna, 1877 - Key West, Florida, 1896).

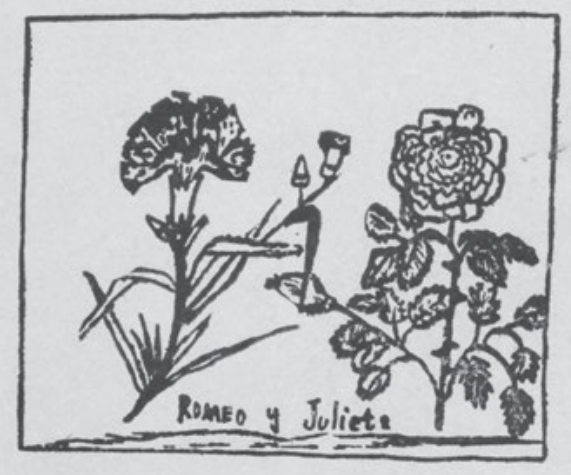

Abb. 85: „El clavel y la rosa“, Zeichnung von Juana Borrero, 1882.

Diese Frage verrät viel über das paradoxe Verhältnis von Leben, Lesen und Lieben, das sich um den imaginierten Mann aufbaut und Juana Borrero bis zu ihrem tragischen Tod im nordamerikanischen Exil im März 1896 verzehrte und nicht mehr loslassen sollte. Wurde Juana Borrero zu einem simplen Opfer der Lektüre in der Nachfolge von Dantes Paolo und Francesca oder Gustave Flauberts Madame Bovary? Fast will es so scheinen, zumal die Nähe zur romantischen Disposition imaginierter Männlichkeit gerade durch einen Vergleich mit der in Puerto Príncipe, dem heutigen Camagüey - auch Juanas Vater, der Lyriker und Arzt Esteban Borrero stammte von dort - geborenen Gertrudis Gómez de Avellaneda deutlich wird. Juana Borrero war nicht nur die Urgroßnichte der uns bereits bekannten Tula; die große kubanische und spanische Dichterin der Romantik war auch eine entscheidende Vorläuferin in Sachen Dichtkunst und weiblicher Emanzipation. Bis in die Liebesmetaphorik hinein verband gewiss vieles die große Vertreterin 
und Hoffnung des „primer modernismo entre nosotros“25 mit der Lyrikerin des Romanticismo; doch hatten sich trotz aller Kontinuitäten die ästhetischen Parameter zwischen Juana und Gertrudis im Verlaufe des 19. Jahrhunderts geändert.

Denn so, wie sich der von ihr erträumte Mann Carlos Pío Uhrbach zumindest über einen langen Zeitraum bemühte, dem auf ihn projizierten Männerbild eines Nicht-Mannes zu entsprechen, so sah Juana Borrero ihr eigenes Bild und mehr noch ihr eigenes Schicksal durch ein Gedicht vorgeformt. Letzteres hatte einer der großen Dichter des kubanischen Modernismo, Julián del Casal, unter dem Titel Virgen triste veröffentlicht. Juana hatte sich in den kubanischen Barden buchstäblich verknallt; und seine Strophe wurde ihr zur Weissagung eines frühen Todes: „weil ich in Dir bereits die Trauer sehe / Jener Wesen, die früh schon sterben müssen!“26

Erfüllte sich das Verliebt-Sein in den noch jungen Dichter Casal auch nicht, der im Übrigen 1893 kaum dreißigjährig verstarb, so hielt der für seine modernistischen Gesellschaftschroniken berühmte Schriftsteller doch ein wesentliches Element der jungen und zutiefst rebellischen Dichterin fest. Denn aller Erfolg werde Juana Borrero niemals von ihrem „más absoluto desprecio“ und der „más profunda indiferencia“ gegenüber den „opiniones de los burgueses de las letras“ 27 abbringen. Nein, für die Bourgeois der Literatur hatte Juana stets nur abgrundtiefe Verachtung übrig - wie auch für die genderspezifischen Rollenzuweisungen, welche eine patriarchalisch ausgerichtete Kolonialgesellschaft den Frauen zuwies; dagegen begehrte sie auf!

Es ist faszinierend $\mathrm{zu}$ beobachten, wie Juana Borrero als Zeichnerin und Malerin für sich ständig neue Rollen erfand und sich bisweilen als Heilige und Nonne, bisweilen als Prinzessin, bisweilen aber auch als Femme fatale zeichnete. ${ }^{28}$ Immer wieder tauchen auch in ihren Gemälden klischeeartige Darstellungen von Mädchen- und Frauenrollen auf, gegen die sie dezidiert andere Darstellungen nicht-bürgerlicher Existenzen stellte. ${ }^{29}$ In einem grundlegenden Sinne reflektierte sie Geschlechterrollen, die sie als Frau hätte annehmen müssen, denen sie sich aber entschlossen verweigerte. In eine nur bürgerliche Kunstauffassung, in eine nur bürgerliche Lebensauffassung ließ sich ihr Lebensprojekt

25 Vitier, Cintio: Las cartas de amor de Juana Borrero. In: Borrero, Juana: Epistolario. Bd. I, S. 31. 26 Casal, Julián del: Poesías. Edición del Centenario. La Habana: Consejo Nacional de Cultura 1963, S. 189: „porque en ti veo ya la tristeza / de los seres que deben morir temprano!“

27 Ebda., S. 271.

28 Vgl. hierzu Ette, Ottmar: Juana Borrero: convivencia y transvivencia. In: Rodríguez Gutiérrez, Milena (Hg.): Casa en la que nunca he sido extraña. Las poetas hispanoamericanas: identidades, feminismos, poéticas (Siglos XIX - XXI). New York - Bern - Frankfurt am Main: Peter Lang 2017, S. 268-307.

29 Vgl. ebda. 
nicht einzwängen. Und so wurde sie zu der allzu jung verstorbenen, großen weiblichen Stimme im hispanoamerikanischen Modernismo, hinter dessen großen (Männer-) Figuren sie freilich zu Lebzeiten zurückstand.

Jenseits der Dreiecksbeziehung zwischen Lieben, Leben und Lesen, die ich in meiner früheren Vorlesung über LiebeLesen bereits bearbeitet habe, ergeben sich im reichen Schaffen der kubanischen Dichterin viele Anhaltspunkte für ein ständiges Wechseln ihrer Rollen - „ut pictura poesis“, in der Malerei wie in der Literatur. So signierte sie ihre überwältigenden Liebesbriefe an Carlos Pío Uhrbach bisweilen mit ihrem eigenen Namen, bisweilen mit „Carlota“ oder „Desdemona“ und häufiger noch mit „Yvone“, der Protagonistin eines romantischen Gedichts des Kolumbianers Abraham Z. López Penha. Hinter Carlos Pío Uhrbach, einem Nachfolger Casals in der Ästhetik eines kubanischen Modernismo, erscheint immer wieder das Bild des 1893 verstorbenen Dichters; und Juana Borrero wird in ihren Briefen nicht müde zu betonen, sie habe Carlos schon lange bevor sie ihn kennenlernte geliebt und ihn auch bewusst gewählt. ${ }^{30}$ Getreu ihrem dem Tagebuch anvertrauten Schwur hatte sie sich ihren Liebhaber gewählt, nicht umgekehrt: Sie allein zeichnete für diese Liebesbeziehung verantwortlich! Die Liebe geht hier einen Bund mit weiblicher Revolte und Rebellion ein.

Das Leben wird mit Literatur und die Literatur mit Leben randvoll gefüllt: Daraus destilliert Juana Liebe. Die Substanz des Lebens selbst wird in Literatur verflüssigt, indem sie ihrem Carlos in absoluter Radikalität einen nicht mit (einer ,literarischen') Tinte, sondern mit ihrem eigenen (,lebendigen') Blut - „en esta clase de tinta que te sugerirá la mitad de mis pensamientos“"31 - geschriebenen Brief zu lesen gibt, in welchem sie den Briefempfänger vor eine Lebens- und Liebesalternative stellt:

Auf die eine oder andere Art, ist etwa das Vaterland, la Patria, nicht eine Rivalin wie jede andere auch? Und eine glückliche Rivalin, denn Du opferst mich ihr! Es scheint Dir schmachvoll, ihrem Rufe nicht zu folgen ... und es scheint Dir nicht verbrecherisch, mit einem einzigen Schlage alle Hoffnungen einer Seele wie der meinigen zu zerstören? Wenn Dich meine Tränen nicht bewegen, wenn bei Dir die Gewissheit meines Todes nicht den Ausschlag gibt, woraus ist dann Dein Herz gemacht? Entweder Dein Vaterland oder Deine Juana: Wähle Du. Wenn Du gehst, verlierst Du mich. ${ }^{32}$

30 Diese oftmals in ihren Briefen in unzähligen Variationen betonte und reflektierte Tatsache erscheint bisweilen mit drohendem Unterton: „Ahora en el silencio de mi habitación, a solas conmigo misma, comprendo jay! que te quiero más de lo que tú sospechas! Y, hace tiempo! Porque tú sabes muy bien que en esta historia yo fui quien tomé la iniciativa de quererte.“ Borrero, Juana: Epistolario, Bd. I, S. 138.

31 Borrero, Juana: Epistolario, Bd. II, S. 256.

32 Ebda., S. 257 (Interpunktion und Schreibweise im Original). 


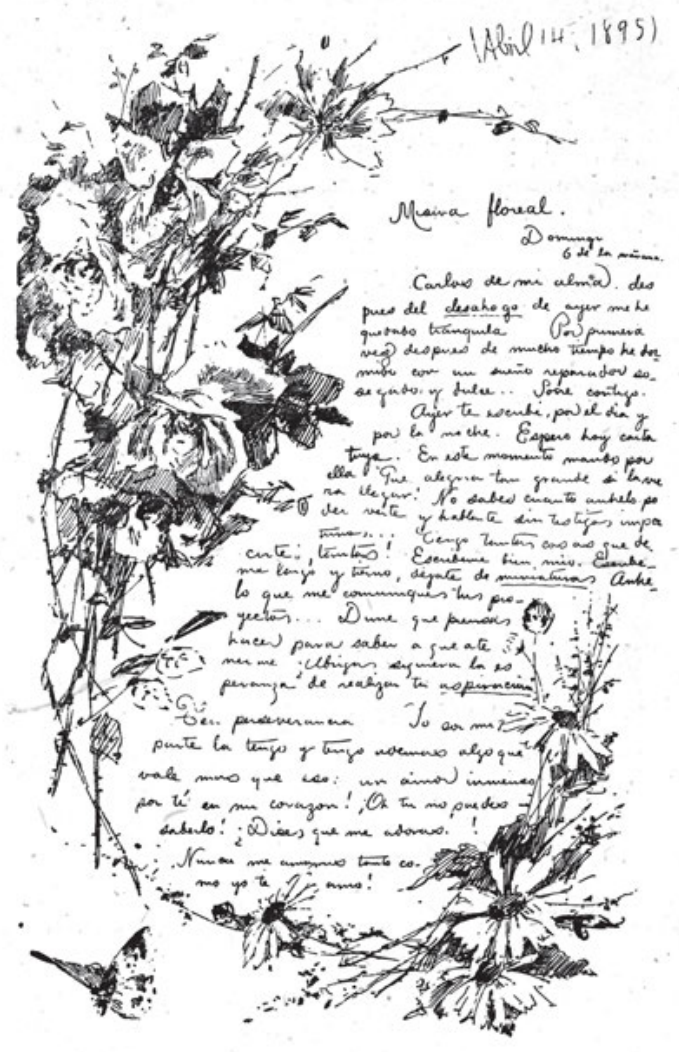

Abb. 86: „Misiva floreal“, Brief von Juana Borrero an Carlos Pío Uhrbach, 14. April 1895.

Juana trägt bewusst den Namen, den ihrer Insel einst Christoph Kolumbus 1492 gab: Juana ist Kuba. Und doch ist Kuba die Rivalin, da ihrer Befreiung vom spanischen Joch jener Krieg gilt, den José Martí gerade entfesselt hatte. Carlos Pío Urbach will sich der Guerra de Martí anschließen, um die „Patria“ zu befreien; ein Unabhängigkeitskampf, in welchem er sein Leben aufs Spiel setzen sollte. Um der Bedrohung des eigenen Lebens durch das bedrohte Leben des Anderen $\mathrm{zu}$ entgehen, verflüssigt in diesem blutroten Brief vom 11. Januar 1896 die Literatin kraft ihrer Liebe das eigene Leben in einer äußersten Anstrengung, die das Risiko eingeht, „mit einem einzigen Schlag“ Leben, Liebe und Lesen für immer zu liquidieren. Doch das Leben schrieb diesmal die Geschichte anders; denn Juana sollte als erste im Exil sterben, bevor noch Carlos Pío, der sich schließlich den aufständischen Truppen anschloss, am Weihnachtstag des folgenden Jahres - 
wie lange vor ihm Martí, den Juana in Begleitung ihres Vaters bei einer Velada in New York kennengelernt hatte - im Kampf fiel. Hatte sie damals in New York schon gespürt, dass von diesem Dichter und Redner, dass von diesem ,Vater` des Modernismus, eine für sie tödliche Gefahr ausging? Jedenfalls erlebten die großen Figuren der kubanischen Lyrik die Jahrhundertwende - und damit die Gründung der Republik - nicht mehr. Es gibt ein Schweigen in der kubanischen Geschichte und Kultur, das wir vom Anfang des 20. Jahrhunderts an nicht länger überhören können; ein Schweigen, das noch heute nachhallt.

In Juana Borrero kulminiert die erkaltete Herzensschrift der Romantik und besetzt die dichterischen Übergänge zwischen Romantik und Modernismus. Diese bilden keine Gegensätze, denn der Modernismo ist in vielerlei Hinsicht die Weiterentwicklung des Romanticismo. Das von Juana Borrero verschriftlichte Herzblut stellt eine nochmalige Radikalisierung eines körperlichen Schreibens mit einer anderen Körperflüssigkeit, jener der Tränen dar, welche bis heute sichtbar das Briefpapier der kubanischen Künstlerin punktieren. Eine Grenze ist hier überschritten, die lebensgefährlich ist für den Lesenden wie für die Liebende und (noch) Lebende, wird damit doch ihr Blut aus dem Kreislauf herausgepresst, um in der Schrift zu erstarren. Und Juana Borrero wurde zur Schrift!

Wir sind am Ende unseres kurzen Durchganges durch das Schreiben jener großen und so jung verstorbenen Modernistin angelangt, die auf die wunderbare Blüte weiblicher Lyrik im spanischen Amerika, auf Alfonsina Storni, auf Juana de Ibarbourou und auf die erste lateinamerikanische Trägerin des Literaturnobelpreises, die Chilenin Gabriela Mistral, vorauswies. ${ }^{33}$ An dieser Stelle mag als der vielleicht dichteste Ausdruck ihrer Kunst jenes Gedicht an Apoll stehen, das noch in der Romantik des 19. Jahrhunderts wurzelt, aber bereits weit über seine EigenZeit hinaus auf jene Schriftstellerinnen deutet, welche die gängigen Stereotypen der Geschlechtertrennung durchbrachen und in der Literatur wie im Leben neue Maßstäbe setzten. Juana Borrero starb zu jung, als dass sie ein eigenes ModerneProjekt hätte entwickeln können; doch war eines gewiss: Nicht dem weiblichen Verlangen, sondern dem männlichen So-Sein mussten Zügel angelegt, ja der männliche Wille zur Macht gebrochen werden. In ihrem dionysischen Sonett an Gott Apoll wird der Mann gleichsam fest-gestellt:

\footnotetext{
Marmorn, stolz und strahlend schön,

Krönung des Gesichts die Süße,

Um die Stirne fall'n die Grüße

Seiner Haare Locken wunderschön.
}

33 Vgl. zu diesen drei herausragenden Lyrikerinnen Ette, Ottmar: Von den historischen Avantgarden bis nach der Postmoderne, S. 423-479. 
Schling' meine Arm' ich, seinen Hals verwöhnen,

Und umfass ich seine hellste Blöße,

Strebend nach Glück, Geschick und Größe,

Auf seiner Stirne meiner Lippen Küsse ertönen.

Mich an die unbeweglich' Brust anschmiegend

Bet' ich der Schönheit Ungerührtheit an,

Will sie beleben, verzweifelt, wie ich kann,

Bin wie geführt in meinem Wahn von Amor,

Ließ meiner zarten Küsse Brennen ich fortan

Auf seinem Körper, auf dem kalten Marmor! ${ }^{34}$

Marmóreo, altivo, refulgente y bello,

Corona de su rostro la dulzura,

Cayendo en torno de su frente pura

En ondulados rizos sus cabellos.

Al enlazar mis brazos a su cuello

Y al estrechar su espléndida hermosura

Anhelante de dicha y de ventura

La blanca frente con mis labios sello.

Contra su pecho inmóvil, apretada

Adoré su belleza indiferente,

$\mathrm{Y}$ al quererla animar, desesperada,

Llevada por mi amante desvarío,

Dejé mil besos de ternura ardiente

Allí apagados sobre el mármol frío!

34 Borrero, Juana: Apolo. In (dies.): Poesías. La Habana: Academia de Ciencias de Cuba 1966, S. 80. 